\title{
Unified explanation for dark matter and electroweak baryogenesis with direct detection and gravitational wave signatures
}

\author{
Mikael Chala, ${ }^{1}$ Germano Nardini, ${ }^{2}$ and Ivan Sobolev ${ }^{3,4}$ \\ ${ }^{1}$ DESY, Notkestrasse 85, D-22607 Hamburg, Germany \\ ${ }^{2}$ Albert Einstein Center, Institute for Theoretical Physics, University of Bern, Sidlerstrasse 5, \\ CH-3012 Bern, Switzerland \\ ${ }^{3}$ Institute for Nuclear Research of the Russian Academy of Sciences, 60th October Anniversary prospect 7a, \\ Moscow 117312, Russia \\ ${ }^{4}$ Department of Particle Physics and Cosmology, Physics Faculty, M. V. Lomonosov Moscow State \\ University, Vorobjevy Gory, 119991 Moscow, Russia
}

(Received 9 June 2016; published 8 September 2016)

\begin{abstract}
A minimal extension of the Standard Model that provides both a dark matter candidate and a strong firstorder electroweak phase transition (EWPT) consists of two additional Lorentz and gauge singlets. In this paper we work out a composite Higgs version of this scenario, based on the coset $S O(7) / S O(6)$. We show that by embedding the elementary fermions in appropriate representations of $S O(7)$, all dominant interactions are described by only three free effective parameters. Within the model dependencies of the embedding, the theory predicts one of the singlets to be stable and responsible for the observed dark matter abundance. At the same time, the second singlet introduces new $C P$-violation phases and triggers a strong first-order EWPT, making electroweak baryogenesis feasible. It turns out that this scenario does not conflict with current observations and it is promising for solving the dark matter and baryon asymmetry puzzles. The tight predictions of the model will be accessible at the forthcoming dark matter direct detection and gravitational wave experiments.
\end{abstract}

DOI: 10.1103/PhysRevD.94.055006

\section{INTRODUCTION}

In light of the celebrated discovery of the Higgs boson $[1,2]$, and the absence of further signatures of new physics at the LHC and other experiments (we cannot assert the real nature of the $750 \mathrm{GeV}$ excess $[3,4]$ for the time being), the success of the Standard Model (SM) of particle physics has become unquestionable nowadays. Nonetheless, there are both experimental observations and theoretical reasons not to consider the SM as a complete description of nature even at low energies. As a matter of fact, the SM does not account for the neutrino oscillations, nor for the evidence of dark matter (DM) and the baryon-antibaryon asymmetry of the Universe, among others. On top of that, the unnatural size of the Higgs mass suggests that new degrees of freedom should show up at energies of the order of the $\mathrm{TeV}$ scale. In this spirit, composite Higgs models (CHMs) [5-7] have played a major role in recent years. In particular, the minimal CHM [8] has deserved special attention, but nonminimal CHMs have also been studied in detail, as their extended scalar sectors may provide solutions to the intricacies of new physics [9-18]. This is especially well motivated, given that departures from the SM predictions can be plausibly hidden in the Higgs sector, which is not precisely measured yet.

Nonminimal CHMs are very predictive in comparison to their renormalizable counterparts built in a bottom-up approach. In CHMs the light scalars are indeed the
pseudo-Nambu-Goldstone bosons (pNGBs) of a spontaneously broken global symmetry of a new strongly coupled sector. The derivative and gauge interactions are hence fixed by the coset structure. The leading-order one-loop potential is instead computed as an expansion on spurion insertions parametrizing the explicit breaking of the global symmetry [19]. The crucial point in this regard is that the number of independent spurion invariants is generally smaller than the number of independent interactions compatible with the remnant symmetry, which in addition may be larger than the SM gauge group. A caveat is still in order for that matter; namely small spurion multiplets are typically disfavored. In fact, they used to generate no quartic term for the Higgs boson at leading order; the proper size of such a term arising only by assuming large next-to-leading order corrections, which tend to make the usual perturbative ordering unreliable [19].

In view of the discussion above, in the present paper we explore the phenomenology of a nonminimal CHM based on the coset $S O(7) / S O(6)$. We show that by taking spurions transforming in the fundamental representation 7 and the symmetric representation 27 of $S O(7)$, the model provides a rather predictive setup where the low-energy scalar sector consists of a Higgs field and two real scalars, $\eta$ and $\kappa$, that are singlets under the SM gauge group. Remarkably, this $S O(7)$ breaking pattern leads to an approximate $Z_{2}$ symmetry for $\kappa$ and an exact $Z_{2}$ symmetry for $\eta$. In addition, the field $\kappa$ does not acquire a vacuum 
expectation value (VEV). It is hence stable and turns out to be a good DM candidate. At the same time, within the (modest) model dependencies of the theory, the singlet $\eta$ acquires a VEV prior to the electroweak symmetry breaking and ultimately promotes a strong first-order electroweak phase transition (EWPT) with sizable gravitational wave signals. Besides, higher-dimensional operators with sizable $C P$-violation phases are expected [11], opening up the possibility of electroweak baryogenesis [20] (related results in noncomposite models are discussed in Refs. [21-24]). Notably, domain wall problems avoided for the $Z_{2}$ symmetry of $\kappa$ is not exact. Hence, within the natural values, the model can potentially solve the hierarchy problem as well as the cosmological evidence of DM and the baryon-antibaryon asymmetry of the Universe. Forthcoming experiments will be able to probe the model predictions.

The rest of this paper is structured as follows. In Sec. II we revisit briefly the basics of CHMs, paying special attention to the computation of the scalar potential. In Sec. III we introduce the model. In Sec. IV we work out the implications for DM while in Sec. V we study the EWPT. In Sec. VI we discuss the current constraints and future probes, including gravitational wave searches, Higgs physics, DM direct-detection experiments, and LHC searches for DM and dijet resonances. Section VII is devoted to conclusions.

\section{A QUICK LOOK AT COMPOSITE MODELS}

In this section we present some introductory ideas on $\mathrm{CHMs}$ and set up conventions. Readers interested in further details are referred to, e.g., the recent review [19], whereas those familiar with CHMs may go directly to Sec. III.

CHMs extend the SM with a new strongly interacting sector characterized by a typical scale $f \sim \mathrm{TeV}$ and a coupling $g$. This new sector is endowed with an approximate global symmetry group, $G$, spontaneously broken into some subgroup, $H$, containing the SM gauge group. The Higgs boson is assumed to be a pNGB of this symmetry-breaking pattern, and hence is naturally lighter than $f$.

The global symmetry is assumed to be explicitly broken mainly by the linear mixings between the SM fermions and composite operators $\mathcal{O}$ of the strong sector. The dynamics of the Higgs and other potential pNGBs is dictated by the following Lagrangian:

$$
\mathcal{L}=\mathcal{L}_{\sigma}+\mathcal{L}_{y}-V
$$

$\mathcal{L}_{\sigma}$ describes the gauge and (derivative) scalar interactions. It is fixed by the coset structure $G / H$. At the leading order in the derivative expansion, it reads

$$
\mathcal{L}_{\sigma}=\frac{f^{2}}{4} \operatorname{Tr}\left(d_{\mu} d^{\mu}\right)
$$

where $d_{\mu}$ is the projection of the Maurer-Cartan one-form $\omega_{\mu}=i U^{-1} D_{\mu} U$ into the broken generators $T^{i}$, with $U$ being the Goldstone matrix

$$
U[\Pi]=\exp \left\{-i \frac{\sqrt{2}}{f} h_{i} T^{i}\right\}
$$

and $h_{i}$ the pNGBs. $\mathcal{L}_{y}$ and $V$ stand for the Yukawa Lagrangian and the scalar potential, respectively. They arise from the explicit breaking of the global symmetry. According to the partial compositeness setup [25], such a breaking is provided by

$$
\mathcal{L}_{\text {mix }}=\sum_{\Psi} \lambda_{\Psi} \bar{\Psi} \mathcal{O}_{\Psi}+\text { H.c. },
$$

with $\Psi$ running over incomplete representations of $G$ (also called spurions) embedding the SM fermions. The composite operators $\mathcal{O}_{\Psi}$ manifest as vectorlike fermions at low energy. These are expected to be much heavier than the SM and the pNBG fields, and hence do not intervene in the phenomenology under study. Thus, we disregard their effects in what follows.

The so-called dressed fields $\Psi^{D}$ can be constructed out of the spurions as

$$
\Psi^{D} \equiv U^{-1} \Psi
$$

In general, $\Psi^{D}$ transform in reducible representations of the unbroken group $H$, namely

$$
\Psi^{D}=\bigoplus \Psi_{m}^{D}
$$

with $m$ running over the irreducible representations of $H$.

The dressed fields are useful to determine the invariants of $H$. By calling $I_{n}^{j}$ the $j$ th invariant of $H$ involving a number $n$ of fields, the scalar potential can be expressed as the following expansion [19]:

$$
V \sim\left(\frac{\lambda_{\Psi}}{g}\right)^{2} \sum_{j} c_{2}^{j} I_{2}^{j}+\left(\frac{\lambda_{\Psi}}{g}\right)^{4} \sum_{j} c_{4}^{j} I_{4}^{j}+\cdots,
$$

where $c_{m}^{j}$ are in practice free parameters. Given that $\lambda_{\Psi}$ is expected to be much smaller than the new strong coupling $g, I_{2}^{j}$ dominate the previous expansion. However, when $\Psi$ transforms in a small representation of $G$, the leading order invariants $I_{2}^{j}$ do not usually generate the Higgs quartic coupling, and hence electroweak symmetry breaking (EWSB) cannot be achieved at the leading order. ${ }^{1}$ Small representations can still be considered if one assumes that $V$ is not dominated by the (formally) leading-order

\footnotetext{
${ }^{1}$ For instance, in the minimal CHM this occurs for the $\mathbf{4}$ and $\mathbf{5}$ representations but not for the $\mathbf{1 4}$ one.
} 
contribution. The problem of this regime is twofold. On one hand, the fine-tuning for keeping the leading-order contribution small is obviously large. On the other hand, sizable next-to-leading order contributions come at the expense of predictivity. Indeed, fewer spurions, and hence free parameters, are present at the leading order. For the sake of example, 2 versus 15 independent spurions arise at the leading and next-to-leading order, respectively, in the $\mathbf{5}$ of $S O(5)$ [19].

In the present work we proceed in the regime where the next-to-leading contributions are subleading, and we hence neglect them unless otherwise stated. As previously discussed, we work out the coset $S O(7) / S O(6)$. We embed the SM fermions in the $\mathbf{7}$ and $\mathbf{2 7}$ of $S O(7)$. The latter arises as the symmetric part of $\mathbf{7} \times \mathbf{7}=1+\mathbf{2 1}+\mathbf{2 7}$, in complete analogy with the 14 in $S O(5)$. Under $S O(6)$, we obtain the following branching rules:

$$
\begin{gathered}
\mathbf{7}=1+\mathbf{6}, \\
\mathbf{2 7}=1+\mathbf{6}+\mathbf{2 0 .} .
\end{gathered}
$$

One and two independent spurion invariants can therefore be constructed at the leading order from the $\mathbf{7}$ and $\mathbf{2 7}$ representations, respectively.

\section{MODEL DESCRIPTION}

The model we analyze is based on the symmetrybreaking pattern $S O(7) \times U(1)^{\prime} / S O(6) \times U(1)^{\prime}$. We proceed in the unitary gauge. Two gauge singlets, $\eta$ and $\kappa$, arise in the pNGB spectrum in addition to the Higgs degrees of freedom $\phi=\left[\phi^{+},\left(h+i \phi^{0}\right) / \sqrt{2}\right] \mathrm{T}$. The addition of a spectator group $U(1)^{\prime}$ is required in order for the SMfermion hypercharges to be correctly reproduced, in the same vein as in the minimal CHM.

The 15 unbroken and 6 broken generators of $S O(7), T$ and $X$, respectively, can be conveniently written as

$T_{i j}^{m n}=-\frac{i}{\sqrt{2}}\left(\delta_{i}^{m} \delta_{j}^{n}-\delta_{i}^{n} \delta_{j}^{m}\right), \quad m<n \in[1,7]$,

$X_{i j}^{m 7}=-\frac{i}{\sqrt{2}}\left(\delta_{i}^{m} \delta_{j}^{7}-\delta_{i}^{7} \delta_{j}^{m}\right), \quad m \in[1,6]$.

The SM $S U(2)_{L} \times U(1)_{Y}$ gauge group is thus generated by

$J_{L}^{1}=\frac{1}{\sqrt{2}}\left(T^{14}+T^{23}\right), \quad J_{L}^{2}=\frac{1}{\sqrt{2}}\left(T^{24}-T^{13}\right)$,

$J_{L}^{3}=\frac{1}{\sqrt{2}}\left(T^{12}+T^{34}\right), \quad J_{R}^{3}=\frac{1}{\sqrt{2}}\left(T^{12}-T^{34}\right)$,

being the hypercharge defined as $Y=J_{R}^{3}+Y^{\prime}$ with $Y^{\prime}$ the generator of $U(1)^{\prime}$.

The dynamics of the pNGBs is described by the Goldstone matrix

$$
U=\exp \left\{-i \frac{\sqrt{2}}{f}\left[T^{47} h+T^{57} \eta+T^{67} \kappa\right]\right\} .
$$

After performing the replacements [9]

$$
\begin{aligned}
& \frac{h^{2}}{h^{2}+\eta^{2}+\kappa^{2}} \sin ^{2}\left\{\left[\frac{h^{2}+\eta^{2}+\kappa^{2}}{f^{2}}\right]^{\frac{1}{2}}\right\} \rightarrow h^{2}, \\
& \frac{\eta^{2}}{h^{2}+\eta^{2}+\kappa^{2}} \sin ^{2}\left\{\left[\frac{h^{2}+\eta^{2}+\kappa^{2}}{f^{2}}\right]^{\frac{1}{2}}\right\} \rightarrow \eta^{2}, \\
& \frac{\kappa^{2}}{h^{2}+\eta^{2}+\kappa^{2}} \sin ^{2}\left\{\left[\frac{h^{2}+\eta^{2}+\kappa^{2}}{f^{2}}\right]^{\frac{1}{2}}\right\} \rightarrow \kappa^{2},
\end{aligned}
$$

we bring $U$ to the two-block matrix form

$U=\left(\begin{array}{ccccc}1_{3 \times 3} & & & & \\ & 1-\frac{h^{2}}{1+\Sigma} & -\frac{h \eta}{1+\Sigma} & -\frac{h \kappa}{1+\Sigma} & h \\ & -\frac{h \eta}{1+\Sigma} & 1-\frac{\eta^{2}}{1+\Sigma} & -\frac{\eta \kappa}{1+\Sigma} & \eta \\ & -\frac{h \kappa}{1+\Sigma} & -\frac{\eta \kappa}{1+\Sigma} & -\frac{\kappa^{2}}{1+\Sigma} & \kappa \\ & -h & -\eta & -\kappa & \Sigma\end{array}\right)$,

where

$$
\Sigma=\sqrt{1-h^{2}-\eta^{2}-\kappa^{2}} .
$$

The sigma model interactions are thus described by the Lagrangian

$$
\mathcal{L}_{\sigma}=\mathcal{L}_{\text {kinetic }}+\frac{1}{2} \frac{\left(h \partial_{\mu} h+\eta \partial_{\mu} \eta+\kappa \partial_{\mu} \kappa\right)^{2}}{f^{2}-h^{2}-\eta^{2}-\kappa^{2}} .
$$

At the level of the sigma model there is a $Z_{2} \times Z_{2} \times Z_{2}$ symmetry given by $h \rightarrow-h, \kappa \rightarrow-\kappa$, and $\eta \rightarrow-\eta$, for the coset is symmetric. This symmetry will be broken only by the external sources, which will also induce a potential for the pNGBs.

At the renormalizable level, the most general potential for $\eta$ (stable), $\kappa$, and the Higgs boson $h$ reads

$$
\begin{aligned}
V= & -\frac{1}{2} \mu_{h}^{2} h^{2}+\frac{1}{2} \mu_{\eta}^{2} \eta^{2}+\frac{1}{2} \mu_{\kappa}^{2} \kappa^{2} \\
& +\frac{1}{3} A_{\kappa h} \kappa h^{2}+\frac{1}{3} A_{\kappa \eta} \kappa \eta^{2}+\frac{1}{3} A_{\kappa} \kappa^{3} \\
& +\frac{1}{4} \lambda_{h} h^{4}+\frac{1}{4} \lambda_{\eta} \eta^{4}+\frac{1}{4} \lambda_{\kappa} \kappa^{4} \\
& +\frac{1}{4} \lambda_{h \eta} h^{2} \eta^{2}+\frac{1}{4} \lambda_{h \kappa} h^{2} \kappa^{2}+\frac{1}{4} \lambda_{\eta \kappa} \eta^{2} \kappa^{2},
\end{aligned}
$$

which involves 12 independent parameters. Not all of them will, however, be generated in the present composite setup, 
at least at the (unsuppressed) leading order. In particular, if we want $\kappa$ to lead to a two-step EWPT and $\eta$ to be a DM candidate without conflicting with Higgs searches (see Secs. IV and V), the following conditions must hold: (i) $\eta \rightarrow-\eta$ is an unbroken symmetry; (ii) $\mu_{\kappa}^{2}<0$; and (iii) the physical masses of $h$ and $\kappa$ are such that $m_{h}<2 m_{\kappa}$, which is favored by $\lambda_{h \kappa} \gtrsim \lambda_{h}$.

In the present composite scenario, a minimal content satisfying the above three conditions consists of the mixing Lagrangian

$$
\mathcal{L}_{\text {mix }}=\sum_{\Psi} \lambda_{\Psi_{R}}{\overline{\Psi_{R}}}^{I}\left(\mathcal{O}_{\Psi}^{R}\right)_{I}+\sum_{\Psi^{\prime}} \lambda_{\Psi_{L}} \bar{\Psi}_{L}^{I}\left(\mathcal{O}_{\Psi}^{L}\right)_{I}+\text { H.c. },
$$

where the first sum extends over $\Psi=T, B, C$ and the second over $\Psi^{\prime}=Q^{t}, Q^{b}, Q^{c}$, with $T_{R}$ and $C_{R}$ transforming in complete singlets of $S O(7)$ with $U(1)^{\prime}$ charge $2 / 3, B_{R}$ and $Q_{L}^{b}$ transforming in fundamental representations 7 of $S O(7)$ with $U(1)^{\prime}$ charges $-1 / 3$, and $Q_{L}^{t}$ and $Q_{L}^{c}$ transforming instead in the symmetric representation 27 of $S O(7)$ that results from the tensor product of fundamental representations, i.e., $\mathbf{7} \times \mathbf{7}=1+\mathbf{2 1}+\mathbf{2 7}$. The most general embedding fulfilling these assignments is explicitly provided by ${ }^{2}$

$$
\begin{aligned}
& B_{R}=\left(\begin{array}{lllllll}
0 & 0 & 0 & 0 & 0 & i \gamma b_{R} & b_{R}
\end{array}\right)^{\mathrm{T}}, \\
& Q_{L}^{b}=\frac{1}{\sqrt{2}}\left(\begin{array}{lllllll}
-i t_{L} & t_{L} & i b_{L} & b_{L} & 0 & 0 & 0
\end{array}\right)^{\mathrm{T}}, \\
& Q_{L}^{t}=\frac{1}{2}\left(\begin{array}{ccccccc}
\mathbf{0}_{6 \times 6} & & & & & & i b_{L} \\
& & & & & b_{L} \\
& & & & i t_{L} \\
& & & & & -t_{L} \\
& & & & & 0 \\
& & & & & & 0 \\
i b_{L} & b_{L} & i t_{L} & -t_{L} & 0 & 0 & 0
\end{array}\right), \\
& Q_{L}^{c}=\frac{1}{2}\left(\begin{array}{ccccccc}
\mathbf{0}_{5 \times 5} & & & & & \zeta s_{L} & i s_{L} \\
& & & & & -i \zeta s_{L} & s_{L} \\
& & & & & \zeta c_{L} & i c_{L} \\
& & & & & i \zeta c_{L} & -c_{L} \\
& & & & & 0 & 0 \\
\zeta s_{L} & -i s_{L} & \zeta c_{L} & i \zeta c_{L} & 0 & 0 & 0 \\
i s_{L} & s_{L} & i c_{L} & -c_{L} & 0 & 0 & 0
\end{array}\right) \text {. }
\end{aligned}
$$

\footnotetext{
${ }^{2}$ See Refs. $[9,10,12,14]$ for $Z_{2}$-preserving embeddings in other models of composite DM.
}

Heavier quarks couple more strongly to the composite sector, and hence $b$ and $c$ contributions to the one-loop potential can be neglected unless they are multiplied by a large $\gamma$ or $\zeta$, respectively. ${ }^{3}$

Note also that the drastically different structure of the lepton sector, and in particular the tiny neutrino masses, suggests that the left-handed leptons do not couple sizably to the new strong dynamics in any way. This could be overcome in particular scenarios [26], which would increase the parameter space of this setup. At any rate, we are just assuming that the main contributions to the potential come from the quark sector.

In this embedding the potential acquires the form

$$
\begin{aligned}
V= & -\frac{1}{2} \mu_{h}^{2} h^{2}+\frac{1}{2} \mu_{\eta}^{2} \eta^{2}+\frac{1}{2} \mu_{\kappa}^{2} \kappa^{2} \\
& +\frac{1}{4} \lambda_{h} h^{4}+\frac{1}{4} \lambda_{\kappa} \kappa^{4}+\frac{1}{4} \lambda_{h \eta} h^{2} \eta^{2}+\frac{1}{4} \lambda_{h \kappa} h^{2} \kappa^{2} .
\end{aligned}
$$

The quartic coupling $\lambda_{\kappa}$ is generated only at the next-toleading order, but it has been introduced since it plays an important role in the EWPT phenomenology. At any rate, it is expected to be much smaller than the other quartic couplings. The rest of the parameters are functions of the dimensionless spurion coefficients $\alpha_{q, i}$, as well as $\gamma$ and $\zeta$,

$$
\begin{gathered}
\mu_{h}^{2}=-\frac{1}{2} f^{2}\left(4 \alpha_{t, 1}-7 \alpha_{t, 2}+\alpha_{c, 2} \zeta^{2}\right), \\
\mu_{\eta}^{2}=-2 \alpha_{t, 2} f^{2}, \\
\mu_{\kappa}^{2}=2 f^{2}\left(\alpha_{b} \gamma^{2}+\alpha_{c, 2} \zeta^{2}-\alpha_{t, 2}\right), \\
\lambda_{h}=4\left(\alpha_{t, 2}-\alpha_{t, 1}\right), \\
\lambda_{h \eta}=4\left(\alpha_{t, 2}-\alpha_{t, 1}\right), \\
\lambda_{h \kappa}=4\left[\alpha_{t, 2}-\alpha_{t, 1}+\left(\alpha_{c, 1}-\alpha_{c, 2}\right) \zeta^{2}\right] .
\end{gathered}
$$

In our analysis we will consider the following two broad parameter regimes depending on the actual values of $\alpha_{c, i}$ : Regime I: $\alpha_{c, 2}=-\alpha_{c, 1}$. This is the most natural scenario since the size of these two coefficients is expected to be similar, and it still allows for $\lambda_{h \kappa} \gtrsim \lambda_{h}$, contrary to the case $\alpha_{c, 2}=\alpha_{c, 1}$.

Regime II: $\left|\alpha_{c, 2}\right| \ll\left|\alpha_{c, 1}\right| \sim\left|\alpha_{t, i} / \zeta^{2}\right|$. As we will see, accounting for the DM relic density observation will completely fix the mass of $\eta$ and its interactions with nuclei in this case. ${ }^{4}$

In both cases, the coefficients $\alpha_{q}^{i}$ as well as $\gamma$ and $\zeta$ can be traded by the measured values of the Higgs VEV

\footnotetext{
${ }^{3}$ If both $\gamma$ and $\zeta$ were zero, $\kappa$ would also be protected by a $Z_{2}$ symmetry.

${ }^{4}$ The case $\left|\alpha_{c, 1}\right| \ll\left|\alpha_{c, 2}\right|$ would be quite similar to Regime I.
} 
$(v \simeq 246 \mathrm{GeV})$ and physical mass (yielding $\lambda \simeq 0.13$ ), and only three free parameters, namely, $f, \mu_{\kappa}^{2}$, and $\lambda_{h \kappa}$. Indeed, in Regime I we obtain

$$
\begin{gathered}
\mu_{\eta}^{2}=\frac{1}{3} f^{2}\left[\frac{7}{4} \lambda_{h}+\frac{1}{4} \lambda_{h \kappa}-4 \lambda_{h} \xi\right], \\
\lambda_{h \eta}=\lambda_{h},
\end{gathered}
$$

while in Regime II we get

$$
\begin{gathered}
\mu_{\eta}^{2}=\frac{2}{3} \lambda_{h} f^{2}(1-2 \xi), \\
\lambda_{h \eta}=\lambda_{h},
\end{gathered}
$$

with $\xi \equiv v^{2} / f^{2}$.

Finally, the Yukawa Lagrangian takes the form

$$
\begin{aligned}
\mathcal{L}_{y}= & -\sum_{q=t, b, c} y_{q} \bar{q} q h\left[1-\frac{1}{f^{2}}\left(h^{2}+\eta^{2}+\kappa^{2}\right)\right]^{\frac{1}{2}} \\
& -i \frac{h}{f} \kappa\left[\gamma y_{b} \bar{b} \gamma_{5} b+\zeta y_{c} \bar{c} \gamma_{5} c\right],
\end{aligned}
$$

with $y_{q}$ the Yukawa couplings.

According to these results, several comments are in order here:

(i) Neither a single cubic coupling nor a quartic term for $\eta$ or a quartic coupling between $\eta$ and $\kappa$ are generated at leading order. At higher orders only the $Z_{2}$ symmetry of $\kappa$ is broken. Therefore, since $\mu_{\eta}^{2}$ is predicted to be positive, ${ }^{5} \eta$ is stable and hence a DM candidate. Finally, $\kappa$ is subject to model dependencies that allow $\mu_{\kappa}^{2}<0 .^{6}$

(ii) The second quark generation has to be included in order for the coupling $\lambda_{h \kappa}$ to be large enough to achieve the hierarchy $m_{h}^{2} / 4<m_{\kappa}^{2}=\mu_{\kappa}^{2}+1 / 2 \lambda_{h \kappa} v^{2}$ between the $h$ and $\kappa$ physical masses. Otherwise the experimental bound on the Higgs decay into nonSM particles [28] would be hard to evade.

(iii) $\gamma$ and $\zeta$ could have a small imaginary part, even in the top sector, without substantially changing the equations above. If this was the case, they could provide a new sizable source of $C P$ violation [11], as required by electroweak baryogenesis. The imaginary part would manifest in the potential as a bunch of $\kappa$-odd terms, making the Higgs mix with $\kappa$ after EWSB. This mixing further introduces electric

\footnotetext{
${ }^{5}$ This is apparent in Regime II from Eq. (32), given the large hierarchy between $f$ and $v$, and hence the small value of $\xi$. In Regime I, instead, it could be violated if $-\lambda_{h \kappa} \gg \lambda_{h}$, which is in conflict with Higgs searches, as commented in (ii).

${ }^{6}$ Relevant comments in this respect have been previously pointed out in Ref. [27].
}

dipole moments (EDM), mainly via two-loop diagrams, as discussed in Ref. [11]. According to it, EDMs are under control provided $\kappa$ is close in mass to $h$. This holds even for large values of the imaginary part, which we explicitly disregard.

(iv) From the EWSB conditions one obtains $\left|\alpha_{t, i}\right| \sim$ $\lambda_{h} \simeq 0.13$. Besides we expect $\left|\alpha_{c}\right|<\left|\alpha_{b}\right|<\left|\alpha_{t, i}\right|$. We have checked that, in both regimes, independently of $f \leq 5 \mathrm{TeV}$, any value of $m_{\kappa}$ below $200 \mathrm{GeV}$ and any value of $\lambda_{h \kappa}$ between 0.1 and 0.4 can be reached by barring this parameter space region with mild values of $\gamma, \zeta \in[3,5]$.

\section{DARK MATTER PREDICTIONS}

As previously discussed, $\eta$ can provide a DM candidate since it is protected by a $Z_{2}$ symmetry not even spontaneously broken. The main diagrams contributing to the annihilation of $\eta$ are shown in Fig. 1. For the choice $f \sim$ $\mathrm{TeV}$ that is favored by electroweak precision data and Higgs physics (see Sec. VI), all depicted processes are kinematically accessible. Indeed, as Eqs. (30) and (32) show, the physical mass of $\eta, m_{\eta}$, is larger than the electroweak scale (and thus larger than the quark and remaining pNGB masses) when $f \gg v$. Because of this hierarchy, the DM phenomenology is dominated by one single scale, $m_{\eta}$ (or equivalently $f$ ). No strong dependence on $m_{\kappa}$ is thus expected.

One can use dimensional analysis to estimate the annihilation cross sections of $\eta$. As the processes with mediators are suppressed, from Eqs. (18), (23), and (34) one deduces

$$
\begin{gathered}
\sigma(\eta \eta \rightarrow h h) v_{0} \sim \frac{1}{m_{\eta}^{2}}\left[\lambda_{h}-\frac{4 m_{\eta}^{2}}{f^{2}}\right]^{2}, \\
\sigma(\eta \eta \rightarrow \kappa \kappa) v_{0} \sim \frac{1}{m_{\eta}^{2}}\left[\frac{4 m_{\eta}^{2}}{f^{2}}\right]^{2}, \\
\sigma(\eta \eta \rightarrow t \bar{t}) v_{0} \sim \frac{1}{m_{\eta}^{2}}\left[\frac{m_{t} m_{\eta}}{f^{2}}\right]^{2},
\end{gathered}
$$

with $v_{0}$ the (small) velocity of the colliding DM particles. It is then expected that the $\eta \eta \rightarrow \kappa \kappa$ channel dominates the
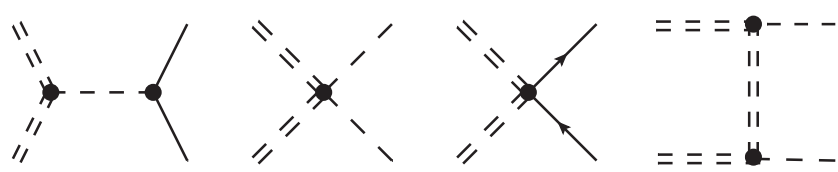

FIG. 1. Main diagrams contributing to the DM annihilation. The double dashed lines stand for $\eta$. In the first plot the solid legs account for either SM particles or $\kappa$. In the second diagram the simple dashed lines represent either the Higgs boson or $\kappa$. In the third diagram the fermion lines stand for (mainly) the top quark. In the fourth diagram the simple dashed lines represent the Higgs boson. 
DM constraint

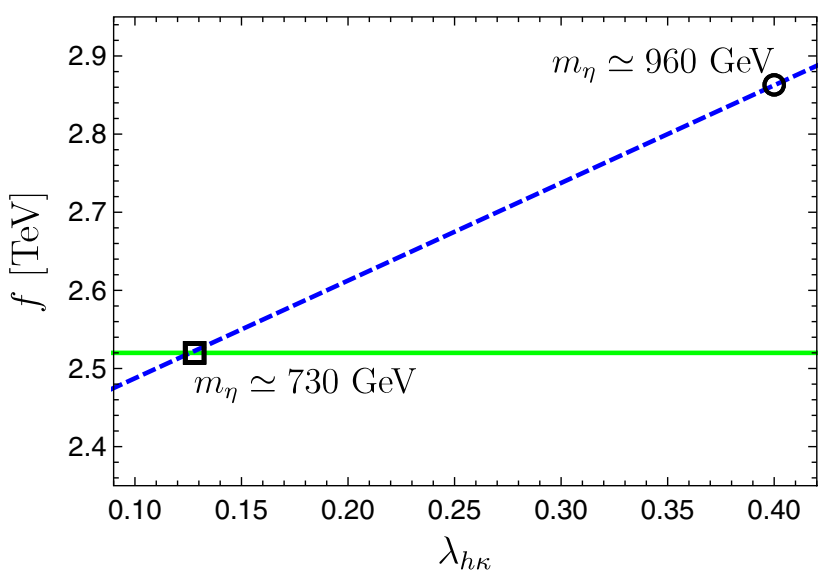

FIG. 2. Value of $f$ leading to $\Omega_{\eta}=\Omega_{\mathrm{DM}}$ as a function of $\lambda_{h \kappa}$ in Regime I (dashed blue line) and Regime II (solid green line). The masses $m_{\eta}$ corresponding to two extreme points are also depicted.

annihilation cross section, for a partial cancellation between the Higgs quadratic coupling and the derivative contribution from $\mathcal{L}_{\sigma}$ arises in $\sigma(\eta \eta \rightarrow h h)$. This implies that the $\mathrm{DM}$ phenomenology can be very different from the smaller composite setup $S O(6) / S O(5)$.

Interestingly, in our framework the DM abundance is fixed by only a few free parameters. Indeed, since $\sigma(\eta \eta \rightarrow$ $\kappa \kappa)$ is the dominant annihilation cross section, the relic density depends only on $\lambda_{h \kappa}$ and $f$ in Regime II, and uniquely on $f$ in Regime II [cf. Eqs. (30) and (32)]. This feature also arises in our numerical study. Specifically, we employ Feynrules [29] to implement the model, and micrOmegas [30] to determine the parameter region where the relic abundance of $\eta, \Omega_{\eta} h^{2}$, is compatible with the experimental measurement $\Omega_{\mathrm{DM}} h^{2}=0.119 \pm 0.003$ [31]. The finding is summarized in Fig. 2 which highlights the constraint $f\left[\lambda_{h \kappa}\right]$ that guarantees $\Omega_{\eta} h^{2}=0.119$ in Regime I (dashed blue line) and Regime II (solid green line). Clearly, for $\lambda_{h \kappa}=\lambda_{h}$ the expected value of $f$ is the same in both regimes. The figure also displays two successful parameter points with somehow extreme values of $\lambda_{h \kappa}$ and the corresponding predictions for $m_{\eta}$. In conclusion, because of the DM relic density constraints, we expect $m_{\eta} \simeq$ $730-960 \mathrm{GeV}$ and $f \simeq 2.4-2.9 \mathrm{TeV}$.

It is worth noting that the rather large $\eta$ mass is in agreement with naturalness arguments. This relies on the fact that, unlike $m_{h}, m_{\eta}$ is not directly related to the tuned $v$, which is indeed (unnaturally) much smaller than the scale $f$ in all composite models. See, for example, Ref. [32] for more details.

\section{ELECTROWEAK BARYOGENESIS}

As generically expected in composite models, and commented above, the interactions between the quarks and the strong sector can contain new $C P$-violation phases. It was observed that these phases, together with the sphaleron processes, can lead to the observed baryon asymmetry, provided the EWPT is of strongly first order [11]. Here we demonstrate that, in our composite model, such a strong EWPT is rather likely within the parameter region compatible with the present constraints, DM bounds included. $^{7}$

We deduce the properties of the EWPT from the oneloop finite-temperature scalar potential $V_{1 \mathrm{~L}}(h, \kappa, \eta ; T)$, with $T$ representing the temperature. Since the new fields have small couplings and negligible mixing with the Higgs, at qualitative level $V_{1 \mathrm{~L}}(h, 0,0 ; T=0)$ is similar to the potential in Eq. (23). Therefore, in the sizable part of the fundamental parameter space predicting $\mu_{\kappa}^{2}<0$ and $\lambda_{h \kappa}>0$, the minima of $V_{1 \mathrm{~L}}(h, \kappa, \eta ; T=0)$ are

$$
\begin{gathered}
v_{1}(T=0)=(v[T=0], 0,0), \\
v_{2}(T=0)=\left(0, v_{\kappa}[T=0], 0\right),
\end{gathered}
$$

with $v_{\kappa}[T=0] \simeq \sqrt{-\mu_{\kappa}^{2} / \lambda_{\kappa}}$ and $v[T=0]=v .^{8}$

This structure of the minima hints at the possibility of a two-step EWPT. In this case the electroweak breaking minimum is reached via the changes of phases $(0,0,0) \rightarrow$ $v_{2}\left(T^{\prime}\right)$ and $v_{2}\left(T_{n}\right) \rightarrow v_{1}\left(T_{n}\right)$, with $T^{\prime}>T_{n}$. The latter transition is the one that is required to be strong (i.e., $\left|v_{1}\left(T_{n}\right)\right| / T_{n}>1$ ) for successful electroweak baryogenesis.

To determine the possible phase transitions and their characteristics, we use CosmoTransitions [41]. In the code we specify $V_{1 \mathrm{~L}}(h, \kappa, \eta ; T)$ in the customary form [42]

$$
V_{1 \mathrm{~L}}(h, \kappa ; T)=V+\Delta V_{\mathrm{CW}}+\Delta V_{T \neq 0},
$$

with $V$ given in Eq. (23) and

$$
\begin{aligned}
\Delta V_{\mathrm{CW}} & =\frac{1}{64 \pi^{2}} \sum_{i}( \pm 1) n_{i} m_{i}^{4}(h, \kappa)\left[\log \frac{m_{i}^{2}(h, \kappa)}{v^{2}}-c_{i}\right], \\
\Delta V_{T \neq 0} & =\frac{T^{4}}{2 \pi^{2}} \sum_{i}( \pm 1) n_{i} J_{ \pm}\left(\frac{m_{i}^{2}(h, \kappa)}{T^{2}}\right), \\
J_{ \pm}(x) & =\int_{0}^{\infty} d y y\left[1 \mp \exp \left(-\sqrt{x^{2}+y^{2}}\right)\right],
\end{aligned}
$$

\footnotetext{
${ }^{7}$ A phase transition, possibly linked to the baryon asymmetry production $[33,34]$, may also occur during the breaking of the composite strong symmetry [35-40]. Such a transition would modify our results only if, in the present setup, it turned out to be of first order and very supercooled, and with a reheating temperature around or below the electroweak scale, characteristics that may or may not be realized depending on the ultraviolet completion [35,39] and its parameter values [33,34].

${ }^{8}$ It is not restrictive to ignore the other symmetric minima. Moreover, because of the (suppressed) explicit breaking of the $\kappa$ discrete symmetry, only one of the two minima $\pm v_{2}$ will be relevant in the evolution of the Universe [11].
} 
EWPT in Regime I

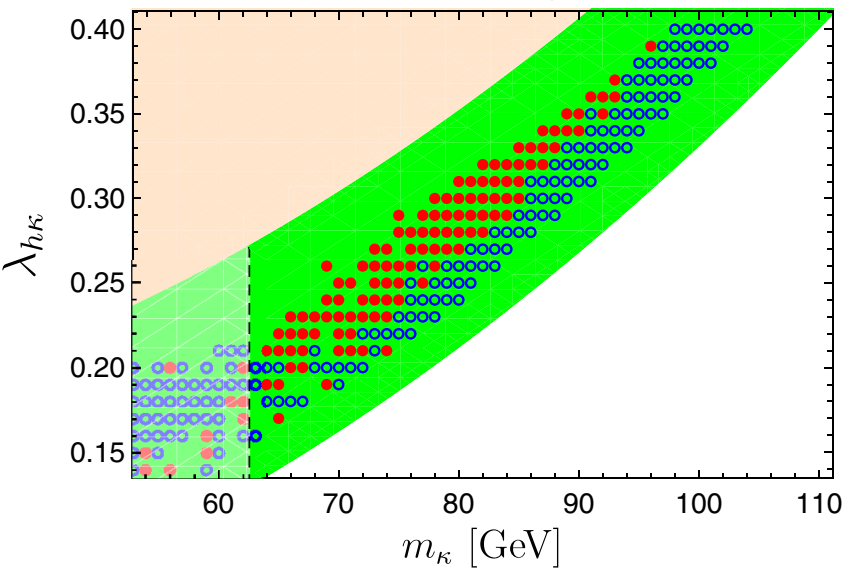

EWPT in Regime II

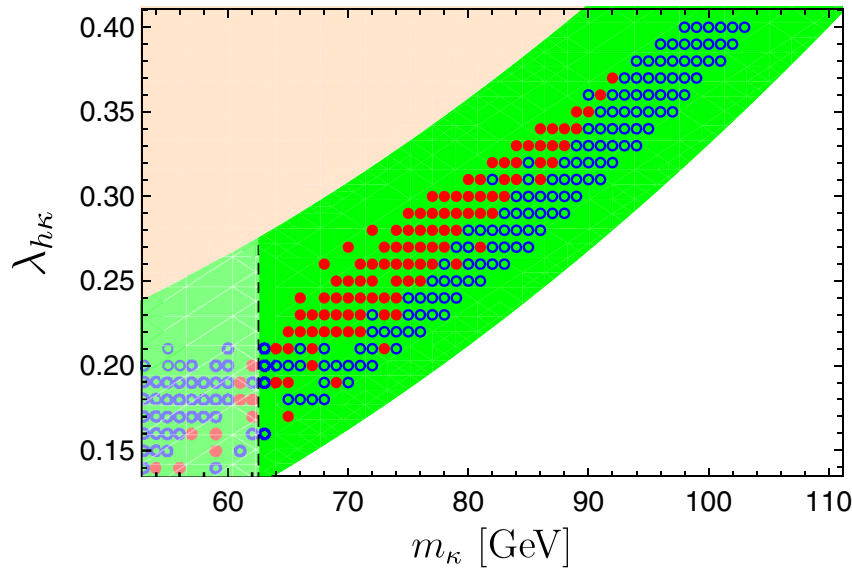

FIG. 3. Scatter plot of the parameter space exhibiting a strong EWPT in Regime I (left panel) and Regime II (right panel). The region in green indicates the points for which $V_{1 \mathrm{~L}}(h, \kappa ; T=0)$ has a local minimum at $v_{1}$ (the contrary in the white area) and such a minimum is deeper than the one at $v_{2}$ (the contrary in the orange area). The points on the left of the black dashed line are unfavored by the Higgs searches. The filled (empty) circles correspond to EWPTs with bubbles expanding (not expanding) at the speed of light. For some parameter points the outcome is not determined because of numerical instabilities in CosmoTransitions, as commented in footnote 9.

in which the dependence on the background field of $\eta$ is removed because $\partial_{\eta}^{2} V>0$ for any $\operatorname{VEV}$ of $h$ and $\kappa$ below $f$. In Eq. (41) $i$ extends to the fields that couple stronger to the Higgs sector, namely the $\kappa-h$ mixing states $\phi_{1,2}$, the SM Goldstones $G^{0, \pm}$, the singlet $\eta$, the top quark $t$, and the gauge bosons $W^{ \pm}$and $Z$. The factor $n_{i}$ is the number of degrees of freedom of the field $i$, and the upper sign (lower sign) in " \pm " and “F” applies to the bosonic (fermionic) contributions. The coefficient $c_{i}$ is equal to $5 / 6$ for gauge bosons and to $3 / 2$ otherwise. Finally, the field-dependent squared masses $m_{\phi_{1}}^{2}(h, \kappa)$ and $m_{\phi_{1}}^{2}(h, \kappa)$ are the eigenvalues of the symmetric matrix $\mathcal{M}^{2}$ with entries

$$
\begin{gathered}
\mathcal{M}_{1,1}^{2}=-\mu_{h}^{2}+3 \lambda_{h} h^{2}+\frac{1}{2} \lambda_{h \kappa} \kappa^{2}, \\
\mathcal{M}_{2,2}^{2}=\mu_{\kappa}^{2}+3 \lambda_{\kappa} \kappa^{2}+\frac{1}{2} \lambda_{h \kappa} h^{2}, \\
\mathcal{M}_{1,2}^{2}=\lambda_{h \kappa} h \kappa,
\end{gathered}
$$

while the other masses are

$$
\begin{gathered}
m_{G^{0, \pm}}^{2}(h, \kappa)=-\mu_{h}^{2}+\lambda_{h} h^{2}+\frac{1}{2} \lambda_{h \kappa} \kappa^{2}, \\
m_{W}^{2}(h, \kappa)=\frac{1}{4} g^{2} h^{2}, \\
m_{Z}^{2}(h, \kappa)=\frac{1}{4}\left(g^{2}+g^{\prime 2}\right) h^{2}, \\
m_{t}^{2}(h, \kappa)=\frac{1}{2} y_{t}^{2} h^{2}, \\
m_{\eta}^{2}(h, \kappa)=C(h),
\end{gathered}
$$

where $g, g^{\prime}$, and $y_{t}$ stand for the $S U(2)_{L}$ and $U(1)_{Y}$ gauge couplings and the top Yukawa, respectively. The function $C(h)$ depends on which regime we consider. Taking into account the DM constraint that establishes the function $f\left[\lambda_{h \kappa}\right]$ (see Fig. 2), we have

$$
C(h)=\frac{1}{3}\left[\frac{7}{4} \lambda_{h}+\frac{1}{4} \lambda_{h \kappa}-4 \lambda_{h} \xi\right] f^{2}\left[\lambda_{h \kappa}\right]+\frac{1}{2} \lambda_{h} h^{2}
$$

in Regime $I$ and

$$
C(h)=\frac{1}{2} \lambda_{h}\left[\frac{4}{3}(1-2 \xi) f^{2}\left[\lambda_{h \kappa}\right]+h^{2}\right]
$$

in Regime II.

For the numerical analysis we scan over the parameter region $\left.\left.\sqrt{-\mu_{\kappa}^{2}} / \mathrm{GeV} \in\right] 0,100\right]$ and $\lambda_{h \kappa} \in[0.1,0.4]$. For each pair $\left\{\mu_{\kappa}^{2}, \lambda_{h \kappa}\right\}$ we consider the lowest value of $\lambda_{\kappa} \in$ $[0.01,0.02, \ldots, 0.06]$ for which the EWPT is strong, if such a transition arises. ${ }^{9}$ The findings are presented in Fig. 3 for Regime I (left panel) and Regime II (right panel). Both filled and empty circles represent the parameter points where the EWPT $v_{2}\left(T_{n}\right) \rightarrow v_{1}\left(T_{n}\right)$ satisfies the condition $v_{1}\left(T_{n}\right) / T_{n}>1$. In the orange area, defined by the condition $V_{1 \mathrm{~L}}\left(v_{1} ; T=0\right) \geq V_{1 \mathrm{~L}}\left(v_{2} ; T=0\right)$ with $\lambda_{\kappa} \leq 0.06$, no EWPT arises because the Universe gets stuck in the phase $v_{2}$. In the white area $\mu_{\kappa}^{2}$ is positive. In the region on the left of the dashed line, all signal strengths of the Higgs into SM particles are diluted, for the channel $h \rightarrow \kappa \kappa$ is allowed. The strong EWPTs found in this area are then ruled out by the

\footnotetext{
${ }^{9}$ Because of some numerical instabilities, the code identifies only a subset of the satisfactory parameter points. Since our aim is to highlight the abundance of points with a strong first-order EWPT, we do try to circumvent this issue and we just display the numerous points that the code finds.
} 
present LHC measurements [28]. Overall, within the parameter space compatible with DM and the EWSB constraints, the ingredients for electroweak baryogenesis are often realized in the present CHM.

We remark that our result is not a full proof that the model can actually reproduce the measured baryon asymmetry [43]. By applying straightforwardly the analysis of Ref. [11] to our setup, one would naively reach a positive conclusion. Nevertheless, the expansion velocities [44-48] of our EWPT bubbles may not be subsonic (i.e., the bubble speed is smaller than $1 / \sqrt{3}$ ) as assumed in Ref. [11]. Were this the case, the evaluation of the baryon asymmetry would be controversial [49]. This particularly applies to the EWPTs marked as filled circles in Fig. 3. They satisfy the runaway condition $\alpha>\alpha_{\infty}$ with

$$
\begin{gathered}
\alpha \simeq \frac{V_{1 \mathrm{~L}}\left(v_{2}\left(T_{n}\right) ; T_{n}\right)-V_{1 \mathrm{~L}}\left(v_{1}\left(T_{n}\right) ; T_{n}\right)}{35 T_{n}^{4}}, \\
\alpha_{\infty} \simeq 4.9 \times 10^{-3}\left(\frac{v_{1}\left(T_{n}\right)}{T_{n}}\right)^{2},
\end{gathered}
$$

which hints at expansion velocities similar to the speed of light $[45,50]$. Consistently, they also fulfill the microphysical-approach runaway condition $\tilde{V}_{1 \mathrm{~L}}\left(v_{1}\left(T_{n}\right) ; T_{n}\right)<$ $\tilde{V}_{1 \mathrm{~L}}\left(v_{2}\left(T_{n}\right) ; T_{n}\right)$, with $\tilde{V}$ being the one-loop thermal potential evaluated in the mean field approximation [51]. ${ }^{10}$ Smaller velocities can instead arise for the EWPTs represented by empty circles (satisfying the nonrunaway relation $\alpha<\alpha_{\infty}$ ), although determining whether such speeds are too large for electroweak baryogenesis would be delicate $[46,48]$. Because of these uncertainties, we cannot go further than claiming that a quantitative explanation of the observed baryon asymmetry is conceivable in the parameter points highlighted in Fig. 3.

\section{OTHER CONSTRAINTS AND FUTURE EXPECTATIONS}

We have seen that, for $f \simeq 2.5-2.9 \mathrm{TeV}, m_{\kappa} \simeq 70$ $120 \mathrm{GeV}, \lambda_{h k} \simeq 0.2-0.4$, and $\lambda_{\kappa} \simeq 0.01-0.06$, which are natural values within our CHM, the observed DM relic abundance and the EWPT that is necessary for electroweak baryogenesis are achieved. In this section we check that these ranges of values are not in conflict with present experimental bounds but testable in the forthcoming years.

\footnotetext{
${ }^{10} \mathrm{We}$ remind the reader that these conditions guarantee a runaway behavior only to those bubbles that have an initial ultrarelativistic speed. Here we adopt the simple criterion $\alpha>\alpha_{\infty}$, where the results are slightly more stringent than the microphysical one, as noticed also in Ref. [52]. On the other hand, unquestionable uncertainties jeopardize these criteria. Further developments in the field are then expected to modify our runaway/nonrunaway classifications.
}
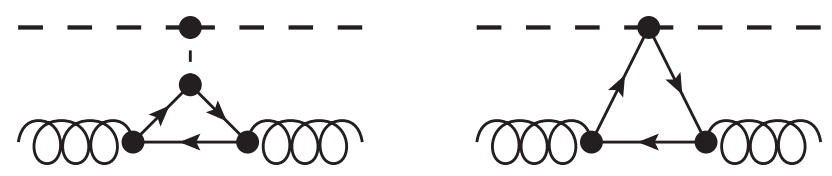

FIG. 4. Main diagrams contributing to the scattering of DM particles by nuclei.

A common prediction to all CHMs (and models with nonlinear realizations of a gauge symmetry in general) is the modification of the Higgs couplings to the SM fermions and gauge bosons. As in the minimal CHM, if we expand Eqs. (18) and (34) to order $\mathcal{O}\left(v^{2} / f^{2}\right)$, we obtain the following ratios of the tree level couplings of the Higgs to two SM particles:

$$
\begin{aligned}
& R_{h V V} \equiv \frac{g_{h V V}}{g_{h V V}^{\mathrm{SM}}} \simeq 1-\frac{v^{2}}{2 f^{2}}, \\
& R_{h \psi \psi} \equiv \frac{g_{h \psi \psi \psi}}{g_{h \psi r \psi}^{\mathrm{SM}}} \simeq 1-\frac{3 v^{2}}{2 f^{2}},
\end{aligned}
$$

where $V$ and $\psi$ stand for any electroweak gauge boson and SM fermion, respectively. Even for $f=2.5 \mathrm{TeV}$, we obtain $R_{h V V} \sim 0.99$ and $R_{h \psi \psi} \sim 0.98$, therefore well within the current LHC limits [28]. Such small deviations from the SM predictions, however, might be accessible at a future linear collider (see, e.g., Ref. [53]).

Concerning direct detection experiments, the two main diagrams contributing to the scattering between DM particles and nuclei are depicted in Fig. 4. The corresponding cross section can be parametrized as [22]

$$
\sigma=\lambda_{h}^{2} \frac{f_{N}^{2}}{4 \pi} \frac{\mu_{r}^{2} m_{n}^{2}}{m_{h}^{4} m_{\eta}^{2}}\left[1+\frac{m_{\eta}^{2}}{f^{2}}\right],
$$

where $m_{n}$ is the nucleon mass, $\mu_{r}$ is the reduced mass of the system (with $m_{\eta} \gg m_{n}$ )

$$
\mu_{r}=\frac{m_{\eta} m_{n}}{m_{\eta}+m_{n}} \sim m_{n} \sim 1 \mathrm{GeV},
$$

and $f_{N} \sim 0.3$ [54-56]. For the considered ranges of parameter values, Eq. (56) yields $\sigma \sim 10^{-46}-10^{-45} \mathrm{~cm}^{2}$, depending on the actual value of $f$. These values are around 1 order of magnitude below the Large Underground Xenon (LUX) experiment bound in the DM mass range $730-960 \mathrm{GeV}$ [57]. However, it will definitely be reachable in the new round of data and experiments [58]. ${ }^{11}$

\footnotetext{
${ }^{11}$ This result can be straightforwardly applied to the simpler model $S O(6) / S O(5)$ with $q_{L}$ transforming in the representation 20, which is analogous to our model in the limit $m_{\kappa} \gg m_{\eta}$. In this case, the observed DM abundance fixes $m_{\eta} \simeq 500 \mathrm{GeV}$, for which the scattering cross section at zero momentum is larger $\left(\sim 2 \times 10^{-45} \mathrm{~cm}^{2}\right)$ but is still below the LUX upper limit.
} 
Regime I and eLISA

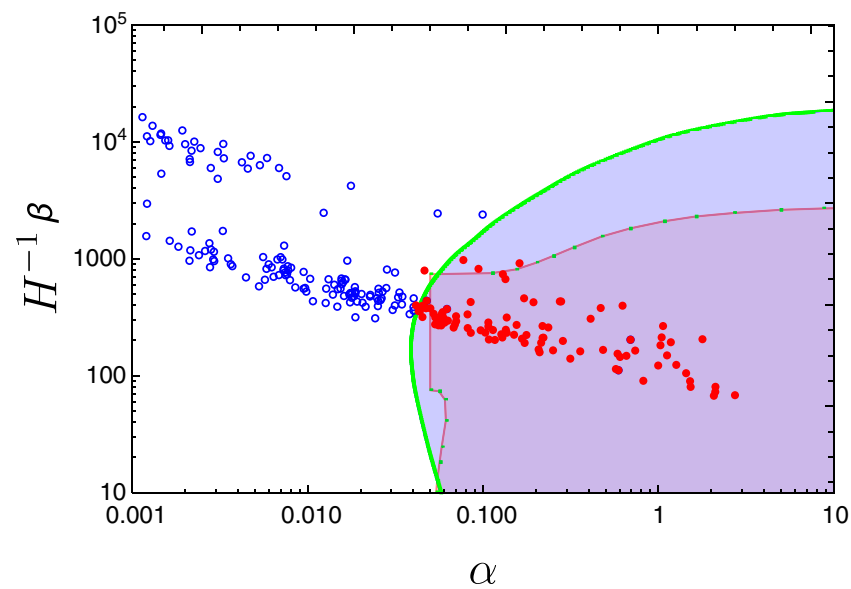

Regime II and eLISA

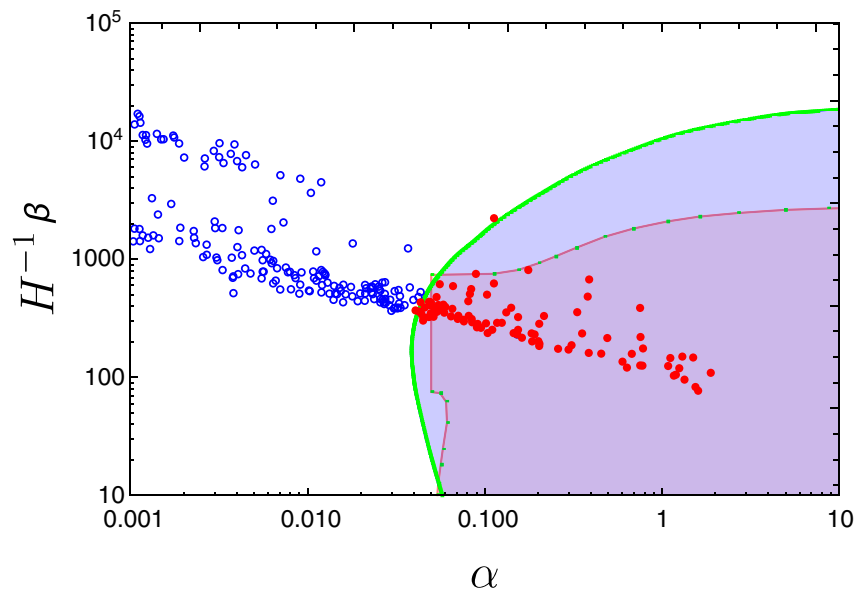

FIG. 5. The identified first-order EWPTs of Regime I (left panel) and Regime II (right panel) in the $\{\alpha, \beta / H\}$ plane. Empty blue circles and filled red circles represent the EWPTs with a nonrunaway and a runaway behavior, respectively. eLISA in the N2A5M5L6 experimental design can test the nonrunaway EWPTs in the (either purple or blue) region on the right of the green curve and the runaway EWPTs in the (purple) region on the right of the red curve.

On another front, LHC searches for DM in monojet and $t \bar{t}$ events produced in association with large missing transverse energy do not provide relevant constraints. Indeed, the cross sections of these signals are suppressed by the small available phase space and the small ratio $v^{2} / f^{2}$. On the same vein, searches for double $\eta$ or $\kappa$ production mediated by an off-shell Higgs boson are extremely challenging [59-63] and hence negligible. Searches for the Higgs boson decaying to non-SM particles [64] instead constrain only the small region on the left of the dashed vertical line in Fig. 3.

A last collider constraint comes from dijet searches. The field $\kappa$ couples linearly to $c$ and $b$ quarks, mainly. Hence, $\kappa$ can be singly produced in hadron colliders, with a subsequent dijet decay. The strongest and updated upper bound on such a process can be found in Ref. [65] (see also Ref. [66]). Given the large QCD background, the main constraints at $\mathcal{O}(100 \mathrm{GeV})$ invariant masses come from UA2 [67] and LHC searches for dijet resonances in association with a $W$ or a $Z$ at $\sqrt{s}=8 \mathrm{TeV}$ [68], the latest being dominant for invariant masses below $\lesssim 200 \mathrm{GeV}$. In this region, the upper limit on the cross section ranges between $\sim 0.1$ and $0.2 \mathrm{pb}$. On the other hand, by using MadGraph [69] we find that, even for $\gamma=\zeta=10$, the production of $\kappa$ in association with a massive gauge boson is smaller than $0.002 \mathrm{pb}$ for any mass in our range of values. The particle $\kappa$ would then be difficult to discover by means of the usual analyses.

Flavor constraints can be evaded provided that, as we assume, the fermion mixings in the infrared are SM-like, whose explanation is a common issue to all realistic models of composite Higgs in which the elementary fermions mix with different operators of the strong sector [19]. This depends strongly on the particularities of the ultraviolet, and therefore goes beyond the scope of this study.

Finally, a strong EWPT produces a gravitational wave stochastic background whose typical spectrum has a peak at frequencies eLISA is sensitive to [70,71]. eLISA might hence be able to probe the CHM under study. We explore this possibility by following Ref. [50], assuming the plausible "N2A5M5L6" eLISA experimental design [50,72]. Thus, for each point in Fig. 3, we calculate $\beta /\left.H \equiv T \partial_{T}\left(S_{3} / T\right)\right|_{T=T_{n}}$ (where $\mathrm{S}_{3}$ is the $\mathrm{O}_{3}$-symmetric bubble action [42]) by running CosmoTransitions. ${ }^{12}$ We display these points in the $\{\alpha, \beta / H\}$ plane, and check whether they are located inside the pertinent eLISA detection region. The findings for Regime I ( Regime II) are presented in the left (right) panel of Fig. 5. The nonrunaway (runaway) strong-EWPT points are displayed as empty blue (filled red) circles. The purple area (the junction of the blue and purple areas) corresponds to the N2A5M5L6 eLISA detection region for runaway scenarios with $T_{n} \approx 50 \mathrm{GeV}$ and $\alpha_{\infty} \approx 0.05$ (for nonrunaway scenarios at $\left.T_{n} \approx 50 \mathrm{GeV}\right) .^{13}$ It results that, among the strong EWPTs identified in Sec. V, none of those with a nonrunaway behavior is detectable, whereas the

\footnotetext{
${ }^{12}$ The code determines $T_{n}$ by solving the condition $S\left(T_{n}\right) / T_{n}=140$ [41]. It is then easy to obtain a second temperature, $T_{C}$, given by $S\left(T_{C}\right) / T_{C}=C$, and use that in the approximation $\beta / H \approx T_{n}(C-140) /\left(T_{C}-T_{n}\right)$. We use $C=240$ in our estimates.

${ }^{13}$ The choice of these particular regions is due to the fact that in our set of points the nucleation temperature of the EWPT is 30$80 \mathrm{GeV}$, and that the points at the border of detection turn out to have $\alpha_{\infty} \simeq 0.05$. The regions are taken from Ref. [50].
} 
runaway ones can be basically all probed. ${ }^{14}$ From Fig. 3 we hence deduce that, overall, eLISA can detect a sizable fraction of the strong EWPTs predicted within our model.

\section{CONCLUSIONS}

We have presented a composite version of the Higgs sector extended with two gauge singlets $\eta$ and $\kappa$ based on the coset $S O(7) / S O(6)$. The embedding of the elementary fermions into appropriate representations of $S O(7)$ can both make $\eta$ stable and generate a negative quadratic term for $\kappa$. As a consequence, this theoretical setup can provide a natural explanation for the observed dark matter abundance and the baryon-antibaryon asymmetry via freeze-out and electroweak baryogenesis. We have emphasized that, contrary to its renormalizable counterpart, the dominant terms in the scalar potential are described by only three free parameters, namely the physical mass $m_{\eta}$ of the dark matter scalar, the physical mass $m_{\kappa}$ of the second singlet, and the coupling $\lambda_{h \kappa}$ of the quartic interaction between the

\footnotetext{
${ }^{14}$ We stress that this sharp conclusion is specific to our model where empirically $\alpha_{\infty} \approx \sqrt{\alpha} / 2$, whence $\alpha \lesssim 0.05$ for all nonrunaway cases (defined by the condition $\alpha<\alpha_{\infty}$ ). This, of course, depends on the adopted runaway condition and its approximation in Eq. (53). For a much more stringent runaway criterion, some of the filled circles would certainly become detectable nonrunaway points.
}

Higgs and $\kappa$. We have shown that fulfilling the dark matter relic density observation requires $m_{\eta} \sim 730-960 \mathrm{GeV}$, irrespective of $m_{\kappa}$, which is required to live in the region 50-100 GeV for $\lambda_{h \kappa} \sim 0.15-0.4$ to trigger the strong first-order electroweak phase transition required by electroweak baryogenesis. We have subsequently studied the implications of this scenario in Higgs physics, dark matter direct detection experiments, LHC searches for dark matter, as well as searches for dijet resonances. All together, they are in agreement with current data. The observation (or not) of a dark matter direct detection signal in the sub-TeV region compatible with a total cross section of $10^{-46}-10^{-45} \mathrm{~cm}^{2}$, possibly followed by the measurement of the gravitational wave stochastic background at eLISA, will definitely shed light on whether this model is realized in nature.

\section{ACKNOWLEDGMENTS}

We are grateful to Thomas Konstandin and Geraldine Servant for useful comments on the manuscript. I. S. thanks the DESY Theory Group for hospitality in the context of the DESY Summer School during the first stage of this work. G. N. thanks the eLISA cosmology working group for useful discussions and M. Codiglia for seminal explanations. The work of G.N. is supported by the Swiss National Science Foundation under Grant No. 200020155935. The work of I. S. is supported by RFBR Grant No. 16-32-00894.
[1] G. Aad et al. (ATLAS Collaboration), Phys. Lett. B 716, 1 (2012).

[2] S. Chatrchyan et al. (CMS Collaboration), Phys. Lett. B 716, 30 (2012).

[3] ATLAS Collaboration, Report No. ATLAS-CONF-2015081.

[4] CMS Collaboration, Report No. CMS-PAS-EXO-15-004.

[5] D. B. Kaplan and H. Georgi, Phys. Lett. B 136B, 183 (1984).

[6] D. B. Kaplan, H. Georgi, and S. Dimopoulos, Phys. Lett. 136B, 187 (1984).

[7] S. Dimopoulos and J. Preskill, Nucl. Phys. B199, 206 (1982).

[8] K. Agashe, R. Contino, and A. Pomarol, Nucl. Phys. B719, 165 (2005).

[9] B. Gripaios, A. Pomarol, F. Riva, and J. Serra, J. High Energy Phys. 04 (2009) 070.

[10] J. Mrazek, A. Pomarol, R. Rattazzi, M. Redi, J. Serra, and A. Wulzer, Nucl. Phys. B853, 1 (2011).

[11] J. R. Espinosa, B. Gripaios, T. Konstandin, and F. Riva, J. Cosmol. Astropart. Phys. 01 (2012) 012.
[12] M. Frigerio, A. Pomarol, F. Riva, and A. Urbano, J. High Energy Phys. 07 (2012) 015.

[13] E. Bertuzzo, T. S. Ray, H. de Sandes, and C. A. Savoy, J. High Energy Phys. 05 (2013) 153.

[14] M. Chala, J. High Energy Phys. 01 (2013) 122.

[15] L. Vecchi, arXiv:1304.4579.

[16] B. Bellazzini, C. Csáki, and J. Serra, Eur. Phys. J. C 74, 2766 (2014).

[17] R. Nevzorov and A. W. Thomas, Phys. Rev. D 92, 075007 (2015).

[18] N. Fonseca, R. Z. Funchal, A. Lessa, and L. LopezHonorez, J. High Energy Phys. 06 (2015) 154.

[19] G. Panico and A. Wulzer, Lect. Notes Phys. 913, 1 (2016).

[20] V. A. Kuzmin, V. A. Rubakov, and M. E. Shaposhnikov, Phys. Lett. 155B, 36 (1985).

[21] J. M. Cline and K. Kainulainen, J. Cosmol. Astropart. Phys. 01 (2013) 012.

[22] J. M. Cline, K. Kainulainen, P. Scott, and C. Weniger, Phys. Rev. D 88, 055025 (2013); 92, 039906 (2015).

[23] S. Profumo, M. J. Ramsey-Musolf, C. L. Wainwright, and P. Winslow, Phys. Rev. D 91, 035018 (2015). 
[24] M. Jiang, L. Bian, W. Huang, and J. Shu, Phys. Rev. D 93, 065032 (2016).

[25] D. B. Kaplan, Nucl. Phys. B 365, 259 (1991).

[26] A. Carmona and F. Goertz, J. High Energy Phys. 05 (2015) 002.

[27] J. Serra, J. High Energy Phys. 09 (2015) 176.

[28] ATLAS and CMS Collaborations, Report No. ATLASCONF-2015-044.

[29] A. Alloul, N. D. Christensen, C. Degrande, C. Duhr, and B. Fuks, Comput. Phys. Commun. 185, 2250 (2014).

[30] G. Blanger, F. Boudjema, A. Pukhov, and A. Semenov, Comput. Phys. Commun. 192, 322 (2015).

[31] P. A. R. Ade et al. (Planck Collaboration), Astron. Astrophys. 571, A16 (2014).

[32] M. Redi and A. Tesi, J. High Energy Phys. 10 (2012) 166.

[33] G. Nardini, M. Quiros, and A. Wulzer, J. High Energy Phys. 09 (2007) 077.

[34] T. Konstandin and G. Servant, J. Cosmol. Astropart. Phys. 07 (2011) 024.

[35] P. Creminelli, A. Nicolis, and R. Rattazzi, J. High Energy Phys. 03 (2002) 051.

[36] L. Randall and G. Servant, J. High Energy Phys. 05 (2007) 054.

[37] T. Konstandin, G. Nardini, and M. Quiros, Phys. Rev. D 82, 083513 (2010).

[38] T. Konstandin and G. Servant, J. Cosmol. Astropart. Phys. 12 (2011) 009.

[39] B. Hassanain, J. March-Russell, and M. Schvellinger, J. High Energy Phys. 10 (2007) 089.

[40] P. Schwaller, Phys. Rev. Lett. 115, 181101 (2015).

[41] C. L. Wainwright, Comput. Phys. Commun. 183, 2006 (2012).

[42] M. Quiros, Helv. Phys. Acta 67, 451 (1994).

[43] P. A. R. Ade et al. (Planck Collaboration), arXiv: 1502.01589 [Astron. Astrophys. (to be published)].

[44] G. D. Moore and T. Prokopec, Phys. Rev. D 52, 7182 (1995).

[45] J. R. Espinosa, T. Konstandin, J. M. No, and G. Servant, J. Cosmol. Astropart. Phys. 06 (2010) 028.

[46] T. Konstandin, G. Nardini, and I. Rues, J. Cosmol. Astropart. Phys. 09 (2014) 028.

[47] A. Megevand, F. A. Membiela, and A. D. Sanchez, J. Cosmol. Astropart. Phys. 03 (2015) 051.
[48] J. Kozaczuk, J. High Energy Phys. 10 (2015) 135.

[49] C. Caprini and J. M. No, J. Cosmol. Astropart. Phys. 01 (2012) 031.

[50] C. Caprini et al., J. Cosmol. Astropart. Phys. 04 (2016) 001.

[51] D. Bodeker and G. D. Moore, J. Cosmol. Astropart. Phys. 05 (2009) 009.

[52] A. Katz and A. Riotto (to be published).

[53] M. Klute, R. Lafaye, T. Plehn, M. Rauch, and D. Zerwas, Europhys. Lett. 101, 51001 (2013).

[54] J. M. Alarcon, J. Martin Camalich, and J. A. Oller, Phys. Rev. D 85, 051503 (2012).

[55] J. M. Alarcon, L. S. Geng, J. Martin Camalich, and J. A. Oller, Phys. Lett. B 730, 342 (2014).

[56] F. Kahlhoefer and J. McDonald, J. Cosmol. Astropart. Phys. 11 (2015) 015.

[57] D. S. Akerib et al. (LUX Collaboration), Phys. Rev. Lett. 112, 091303 (2014).

[58] P. Cushman et al., arXiv:1310.8327.

[59] Y. Mambrini, Phys. Rev. D 84, 115017 (2011).

[60] A. Djouadi, O. Lebedev, Y. Mambrini, and J. Quevillon, Phys. Lett. B 709, 65 (2012).

[61] A. Djouadi, A. Falkowski, Y. Mambrini, and J. Quevillon, Eur. Phys. J. C 73, 2455 (2013).

[62] A. De Simone, G. F. Giudice, and A. Strumia, J. High Energy Phys. 06 (2014) 081.

[63] N. Craig, H. K. Lou, M. McCullough, and A. Thalapillil, J. High Energy Phys. 02 (2016) 127.

[64] G. Aad et al. (ATLAS Collaboration), J. High Energy Phys. 11 (2015) 206.

[65] M. Chala, F. Kahlhoefer, M. McCullough, G. Nardini, and K. Schmidt-Hoberg, J. High Energy Phys. 07 (2015) 089.

[66] B. A. Dobrescu and F. Yu, Phys. Rev. D 88, 035021 (2013); 90, 079901 (2014).

[67] J. Alitti et al. (UA2 Collaboration), Nucl. Phys. B400, 3 (1993).

[68] ATLAS Collaboration, Report No. ATLAS-CONF-2013074.

[69] J. Alwall, R. Frederix, S. Frixione, V. Hirschi, F. Maltoni, O. Mattelaer, H.-S. Shao, T. Stelzer, P. Torrielli, and M. Zaro, J. High Energy Phys. 07 (2014) 079.

[70] P. A. Seoane et al. (eLISA Collaboration), arXiv:1305.5720.

[71] https://www.elisascience.org.

[72] A. Petiteau (to be published). 